\title{
Automatic Traffic Monitoring from Satellite Images Using Artificial Immune System
}

\author{
Mehrad Eslami $^{1}$ and Karim Faez ${ }^{2}$ \\ ${ }^{1}$ Computer Engineering Department, Azad University of Qazvin, Qazvin, Iran \\ Mehrad.Eslami@gmail.com \\ ${ }^{2}$ Electrical Engineering Department, Amirkabir University of Technology, Tehran, Iran \\ kfaez@aut.ac.ir
}

\begin{abstract}
Automatic and intelligence Road traffic monitoring is a new research issue for high resolution satellite imagery application in transportation. One of the results of this research was to control the traffic jam in roads and to recognize the traffic density quickly and accurately. This article presents a new approach for recognizing the vehicle and the road in satellite high-resolution images in non-urban areas. For road recognition, they used feature extraction and image processing techniques like Hough transform, Gradient, and thresholding operation and they presented an artificial immune approach to extract vehicle targets from high resolution panchromatic satellite imagery. The average of results is about 94 percent and it shows that the used procedure has the suitable efficiency.
\end{abstract}

Keywords: Road Extraction, vehicle Detection, Hough transform, Artificial Immune System, Intelligence Traffic monitoring, Satellite images.

\section{Introduction}

With the growth of urban traffic and the necessity to control it, the attention has been paid to intelligent traffic control systems. Nowadays urban traffic is controlled by the cameras which are installed in highways located at a long distance from each other. With the development of technology, this procedure seems so slow and deficient. According to the satellite images considered recently, the existence of an intelligent system for road and vehicle recognition to control road's traffic will be so useful.

Hence, area-wide images of the entire road network are required to complement these selectively acquired data. Since the launch of new optical satellite systems like IKONOS and NAVTEQ, this kind of imagery is available with 0.6-1.0 meter resolution. Vehicles can be observed clearly on these high resolution satellite images. Thus new applications like vehicle detection and traffic monitoring are raising up.

Traffic, road and vehicle recognition in satellite images with very high resolution are very new discussions in machine vision science which makes many projects and other operations depend on it. Main factors having the most influence on the topic is the number of different objects in the image, the amount of their interconnections and the features that can distinguish them from other objects. 
This paper proposes the technique using the artificial immune network concept to extract the vehicle targets and using Hough transform and parallel lines detection to extract roads and then recognize traffic in space imagery. First we detect road and its boundaries, then we start to detect vehicle in it, because after edge detection, only the objects within the boundaries of that road are processed. To do this, extraction of road and vehicle features is necessary. One of the most useful features of the road is that the road appeared in satellite images will usually be referred to as a direct confinement with a different color [7-8]; so the linear feature can be a proper one to detect road confinement. The other feature of roads is the lines drawn on them, these white lines which exist on almost all roads and help a lot in road detection. The lines close to roadsides and in the middle count as one of the proper features. The other feature used is road's color while in high resolution satellite images, distinguishes completely the roads from the roadside. With thresholding, the roads can be distinguished from background so that other operations can be performed on the image. This colorful threshold can be extracted by averaging images existing in the data. After using road's features to detect it, the vehicle will be detected in the roads. After road detection, vehicle detection is easier, because only the objects within the road limits are to be studied.

We used, the technique using the artificial immune network concept to extract the vehicles. The immune system is one of the highly evolved biological information processing systems and is capable to learn and memorize. Many kinds of immune systems have been studied by mathematical methods. In recent years, applications of artificial immune system have been proposed in many engineering problems [9-10]. In this study, we attempt to use them for target recognition. Observed targets are regarded as foreign antigens, and a template is regarded as an antibody. Complementary template matching is considered to be exactly the same as the binding of the Paratope and Epitope. The algorithm implements morphology operations on images to enhance vehicle features. Some of sub-images in the processed images are selected as the vehicle and non-vehicle training samples for antibody learning. The learned template antibodies are tested on real road segments.

\section{Correlated Issues}

Since satellite images with very high resolution include lots of information and elaborations of recognition process, the choice of appropriate features for recognition is one of the main operations in processing and analyzing satellite images.

The studied satellite images with very high resolution in this paper are caught by xerographic satellites. These images have high resolution which makes it easier to extract their features with high accuracy.

These images are fully colored taken in the same distance from Earth. The distance between cameras and the Earth is very important in taking satellite images, because some of the features which have been used have direct relationship with distance and size dimensions, and if the distance changes noticeably, we will need a new features measurement requiring standard modules.

Here it is assumed that the images are taken from a specific distance and resolution. Processing was done on color images in RGB, HSV. It should be considered that these images are taken of the non-urban roads with low probability of crossroads. 

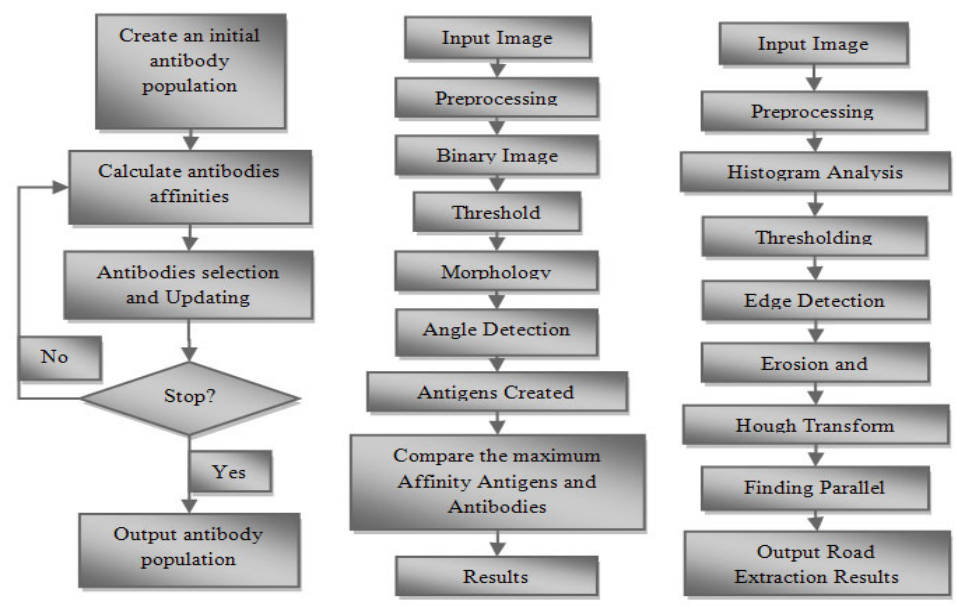

Fig. 1. Left: Antibodies learning flowchart. Middle: Vehicle Detection flowchart. Right: Road Detection Flowchart.

\section{Road Detection}

In this section, the represented method for road detection is implemented on satellite images (Figure 1). To do this, first we applied proper filters of image processing on the image, so that the image's edges will be clearer. One of these filters is sharpening filter which increases the image clearness and makes the image's edges more vivid.

Detection is applied on the image. Road's color is distinguished from other parts of the image with the help of a filter and through thresholding. First, the thresholding is performed to discriminate road from other parts of the image. This operation is done on the base of color difference between the road and other parts of the image.

This thresholding can be measured by the histogram of the road's satellite image. According to the histogram in Figure (2), it can be seen that, this histogram is divided into many areas. The areas which show roads in the image, is ranges 5 to 9 of horizontal axis of histogram which assign the greater amount of image color to itself. According to the histogram of satellite image and analysis of different images values red, green and blue which have the most similarities to the road's background color.
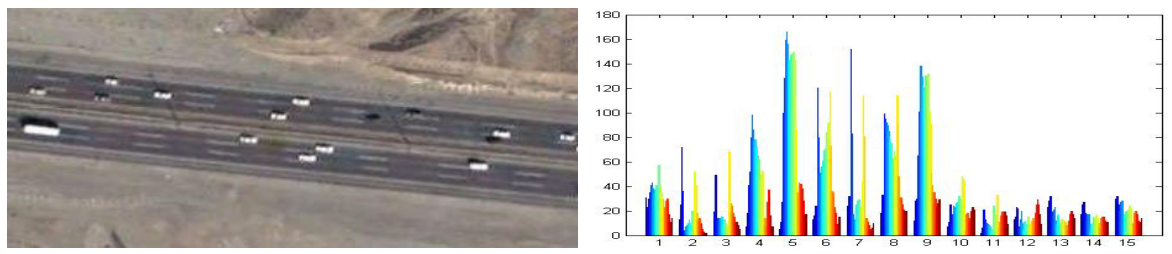

Fig. 2. Left: Satellite image of Karaj-Qazvin Highway. Right: Histogram of Satellite image of a Karaj-Qazvin Highway. 

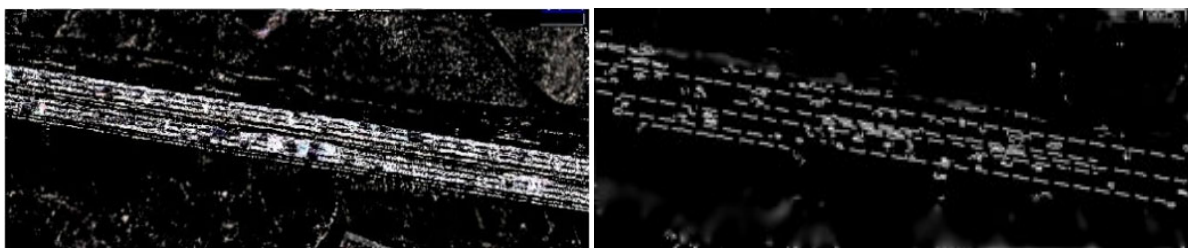

Fig. 3. Left: Road image after thresholding. Right: Road image after canny edge detection operation.

Now the main operation, the road detection, will be done, as it is shown in figures (3) (Road's color-linear of the white lines in sides and the middle of the road).

As it is shown in Figure (3), thresholding has been done, and the spots where the probability of the existence of road is less are in black. So other operations would be more functional. As you can see, there are spots, which are not road, but they are not black and it is because their color is the same as the road and that the spots must be consequently corrected. After thresholding, edge detection will function with better results. For edge detection Canny method will be used. This method has a better efficiency than other methods used in road detection. The result is shown in figure (5).

In digital images where the spots have been shown along with edges, there is a difference in color. So sharpening operations with the use of a filter will increase the color difference in edges and the clearness of the image; also, next operations such as edge detection will be performed with much proper attention. Hough transform is used after edge detection and thresholding in the last phase.

If the point $\left(\mathrm{X}_{\mathrm{i}}, \mathrm{Y}_{\mathrm{i}}\right)$ and equation of a line be like $\mathrm{Y}_{\mathrm{i}}=\mathrm{aX} \mathrm{X}_{\mathrm{i}}+\mathrm{b}$ there are lots of lines coming from $\left(\mathrm{X}_{\mathrm{i}}, \mathrm{Y}_{\mathrm{i}}\right)$ that any of them for the values of $\mathrm{a}, \mathrm{b}$ make the equation $\mathrm{Y}_{\mathrm{i}}=\mathrm{aX} \mathrm{X}_{\mathrm{i}}+\mathrm{b}$. we can recognize with Hough transform the spot that can be in line equation and make a line in the image [13-14, 17].

With the use of Figure (4), all the spots which are located on one line can be obtained. The spot where all curves meet shows a joint line on the specific spots. The spots which are located in parts of the chart that have same angles are parallel lines in the image that show road boundaries.
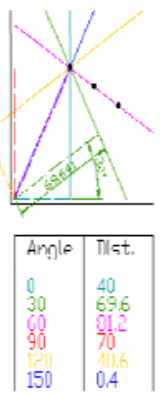
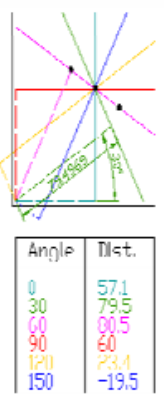

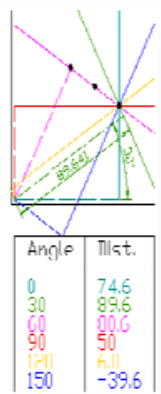

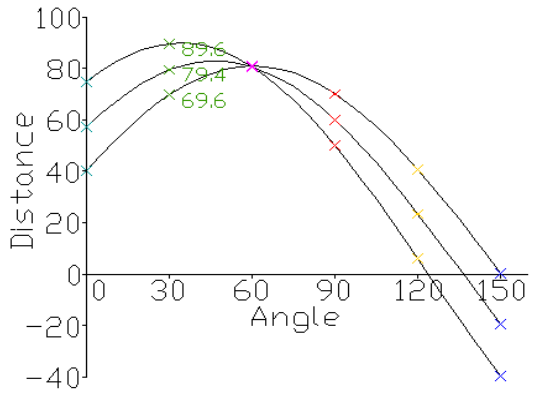

Fig. 4. Left: several lines that crossed a point. Right: the spot where all curves meet make a joint line for all points. 


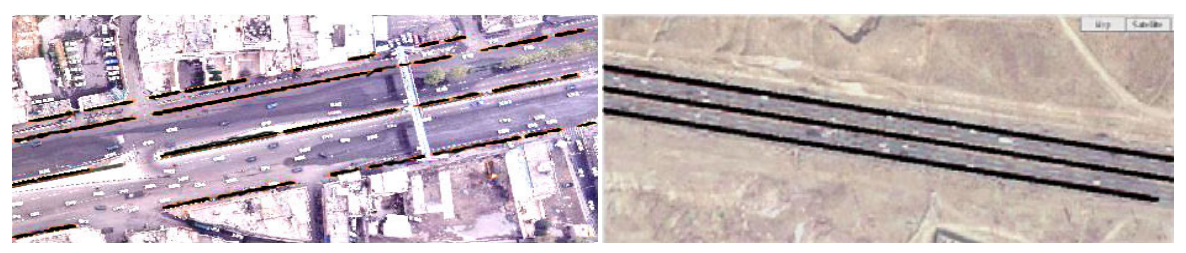

Fig. 5. Road and Highway after detection operation

Hough transform is done with a change of its parameters to improve the efficiency to detect road boundaries, and then vehicle detection in roads will be continued.

\section{Vehicle and Traffic Detection}

\subsection{Definition of Immunological Terms}

In this section, the immunological terms are defined in the following manner:

- Antigen: Vehicle targets.

-Antibody: Vehicle template images extracted from processed images by the morphology transform.

The used morphology transform is to enhance vehicle features. It is defined by

$$
G(f)=f \oplus g-f
$$

Where $g$ is a structuring element, $f$ is a gray scale image, $f \oplus g$ means dilate operation, i.e. Dilation:

$$
(f \oplus g)(x)=\max \left\{f(z) g *(z): z \in D\left[g^{*}\right]\right\}
$$

Where $g_{x}(z)=g(z-x), g^{*}(z)=-g(-z)$ and $\mathrm{D}[g]$ is the domain of $g$.

Figure (6), shows an original image and its morphology processing result. It can be clearly seen that all vehicle bodies or contours are enhanced. These enhanced features can be used to discriminate vehicle targets and non-vehicle targets. Figures (6) and (7) show some antibody examples collected from the morphology processed image, and each example image has same size.

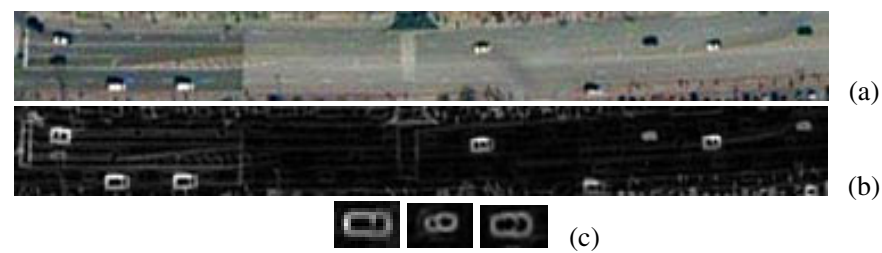

Fig. 6. (a) An original image. (b) The morphology preprocessing result. (c) Antibody examples. 
- Affinity: Matching index. It is inspired from image correlation concept. It is defined by

$$
\mathrm{R}=\frac{\sum_{x=0}^{L-1} \sum_{y=0}^{K-1}(w(x, y)-\bar{w})(f(x, y)-\bar{f})}{\sqrt{\sum_{x=0}^{L-1} \sum_{y=0}^{K-1}(w(x, y)-\bar{w})^{2}} \sqrt{\sum_{x=0}^{L-1} \sum_{y=0}^{K-1}(f(x, y)-\bar{f})^{2}}}
$$

$w(x, y)$ is the template antibody image of size $K$. $L$ and $f(x, y)$ is the antigen image of size $K \cdot L, w$ is the average intensity value of the pixels in template antibody image $\mathrm{w}, f$ is the average intensity value of the pixels in template antigen image $f$.

The greater the value of $R$ has the higher the antibody's affinity.

\subsection{Antibody Learning}

For antibody learning (Figure 1), we setup an image database which includes vehicle samples and non-vehicle samples. In the database, all samples are collected from morphology processed images using same sampling window (Figure 7). To compare Antibodies with Antigens, chosen samples of both of them should be in the same direction. It should be considered that the movement orientation of the vehicles in the road, is the same as road's orientation, which was obtained in the previous section from the equation $\mathrm{Y}=\alpha \mathrm{X}+\beta$. With rotation the vehicles as much as it is desire, all the extracted samples from the roads, will have same orientation. We randomly select $\mathrm{N}$ vehicle samples from the database as the initial antibody population, the rest samples are regarded as training sets. According to the immune network theory, antibodies interact with each other and with the environment (antigens). The interaction property leads to the establishment of a network. When an antibody recognizes an epitope or an idiotope, it can respond either positively or negatively to this recognition signal.

A positive response would result in antibody activation, antibody proliferation and antibody secretion, while a negative response would lead to tolerance and suppression.

According to these antibody properties, we develop an immune network for vehicle detection. A set of rules are proposed for antibody selection and updating in the immune network. These rules are as follows.

Rule 1. Eliminate the antibody if the maximum affinity of the antibody to vehicle samples is under the threshold $(<0.6)$.

Rule 2. Eliminate the antibody that has high similarity over the threshold $(>0.9)$ to other antibodies.

Rule 3. Eliminate the antibody if the affinity of the antibody to any non-vehicle sample is over the threshold $(>0.6)$.

Rule 4. Add a vehicle sample from training sets into the antibody population as a new antibody if the affinity of the vehicle sample to any antibody is under the threshold $(<0.6)$.

Based on above rules, the antibody learning procedure in the immune network is described as follows:

Step 1: Randomly select $\mathrm{N}$ vehicle samples from the database as the initial antibody population. 
Step 2: Evaluate the affinity of each antibody in the population with Eq. (3).

Step 3: Eliminate the antibody according to Rule 1-3.

Step 4: Update antibody population according to Rule 4.

Step 5: Repeat Steps 2-4 until none of antibodies is eliminated and none of new antibodies is added.

Step 6: Save final antibody population for vehicle detection use.

Fig.3 shows the flowchart for antibody learning.

\subsection{Strategy of Detection}

After learning the antibody population, the learned antibody population can be used to detect vehicles in the imagery (Figure 1).

Firstly, according to Eq. (1), implement morphology transform on the original image (Figure 7). Secondly, calculate the maximum affinity to all template antibodies at each pixel point $(i, j)$ by Eq. (3). Thirdly, compare the maximum affinity value $R$ at every point with the given threshold. If the $\mathrm{R}$ is greater than the threshold, the point belongs to a vehicle target and is set as 255 . Otherwise, it belongs to a non-vehicle target and is set as 0. Finally, a post processing based on morphology dilation and erode operations is employed to merge neighborhood vehicle target pixels and locate the center of a vehicle [21].
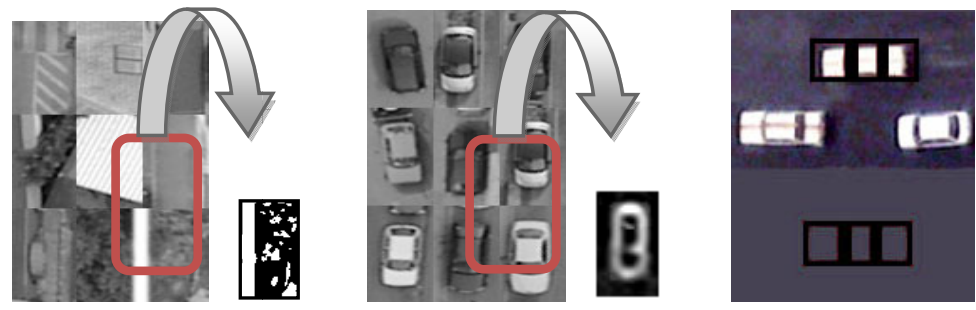

Fig. 7. Left: No-Vehicle, Middle: Vehicle Pattern, Right: Prototype of Vehicle Pattern
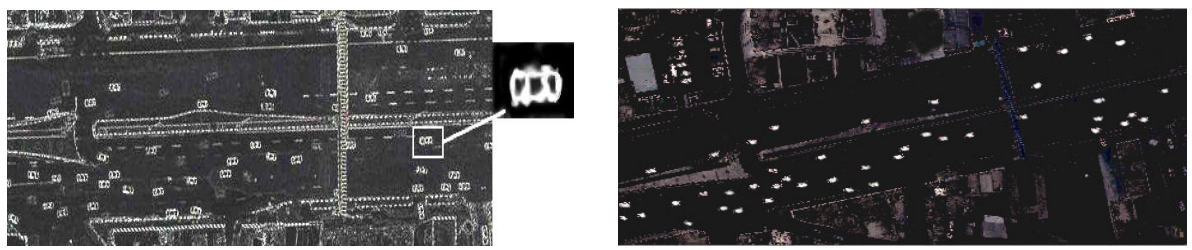

Fig. 8. Left: Morphology operation on image. Right: Vehicles after detection.

Fig. 4 shows the flowchart of the proposed vehicle detection based on the antibody learning.

After vehicle and road detection (Figure 8), we can distinguish the traffic density on the road. Because all images have been photographed from a same distance and resolution and with the number of vehicles in a specific area [12] and the use of thresholding method, we can distinguish the traffic density on the road according to the 
number of vehicles in a specific area and can be sent to the stations. This model also has the same deficiencies. Most important of them is that it cannot distinguish multiple roads in the image.

\section{Results and Conclusions}

In this research, we tried to detect road and vehicle in satellite images with very high resolution. This model can help drivers who want to pass the mentioned road, and also can help policemen to control the traffic on roads. At the present time, these images can be taken by xerography satellite and special airplanes. NAVTEQ panchromatic data set used in our study was collected from Space Imaging Inc. web site [23]. The data set contains different city pictures. A total of 6 roads segments containing over 200 vehicles were collected. Most vehicles in the images are around 8 to 10 pixels in length and around 3 to 5 pixels in width.

Since the vehicles are represented by a short number of pixels, their detection is very sensitive to the surrounding context. Accordingly, the sample database consists of vehicle and non-vehicle samples in a variety of conditions, such as road intersections, curved and straight roads, roads with lane markings, road surface discontinuity, pavement material changes, trees' shadow on the roads, etc. This represents most of the typical and difficult situations for vehicle detection.

Table 1. Results of present method

\begin{tabular}{||c|c|c|c|c|c|c||}
\hline $\begin{array}{c}\text { No. of } \\
\text { vehicles }\end{array}$ & $\begin{array}{c}\text { No. of detected } \\
\text { vehicles }\end{array}$ & $\begin{array}{c}\text { No. of } \\
\text { missing } \\
\text { vehicles }\end{array}$ & $\begin{array}{c}\text { No. of } \\
\text { false alarm }\end{array}$ & $\begin{array}{c}\text { Missing } \\
\text { detection } \\
\text { rate } \%\end{array}$ & $\begin{array}{c}\text { False detection } \\
\text { rate } \%\end{array}$ \\
\hline Road1 & 5 & 5 & 0 & 0 & 0 & 0 \\
\hline Road2 & 8 & 8 & 0 & 0 & 0 & 0 \\
\hline Road3 & 10 & 10 & 0 & 1 & 0 & 10 \\
\hline Road4 & 15 & 15 & 0 & 1 & 0 & 6 \\
\hline Road5 & 19 & 17 & 1 & 1 & 5.3 & 5.2 \\
\hline Road6 & 16 & 15 & 1 & 0 & 6.2 & 0 \\
\hline Road7 & 23 & 23 & 2 & 2 & 8 & 8 \\
\hline Road8 & 60 & 56 & 6 & 5 & 10 & 8.3 \\
\hline Road9 & 52 & 47 & 5 & 4 & 11.5 & 7.6 \\
\hline
\end{tabular}

For each selected image, roads were extracted in advance and vehicle detection was performed only on the extracted road surfaces. To build the vehicle example database, manually delineated the rectangular outer boundaries of vehicles in the image [15-16]. A total of 200 vehicles were delineated in this manner from 5 road segments. Sub-images of $10 \times 5$ pixels centered at vehicle were built in. In addition, 200 non-vehicle sub-image samples covering different road surfaces were also collected to build the non-vehicle example database (Figure (7)). The vehicle example database and used for features extraction. Results are shown in table (1).

Kuthadi Sumalatha, in his master's thesis "Detection of Objects from HighResolution Satellite Images" has worked on road, vehicle and urban areas. He had used thresholding and color extraction methods in his thesis [20] (Figure (9)). 
According to the results obtained, Kuthadi had carried out his research with higher accuracy in comparison with others. With the help of four images which Kutadi used for his work, we compared Kuthadi's method with the recommended method. This comparison is shown in table (2).
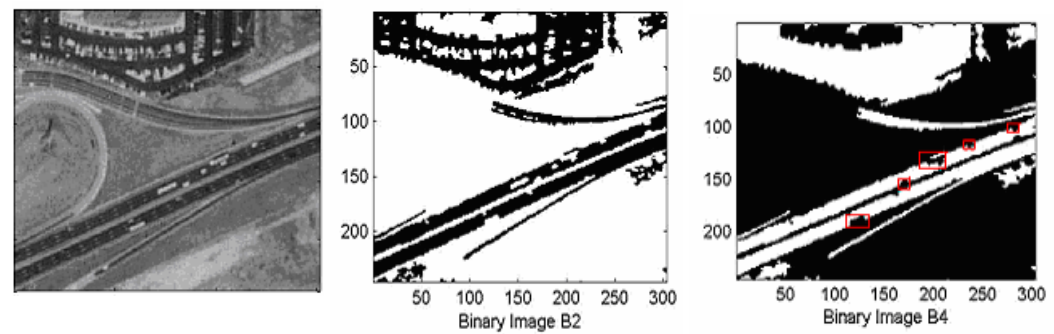

Fig. 9. Kuthadi's result for road and vehicle detection (A highway in France). Left: original image, Middle: road detection, Right: vehicle detection.

Table 2. Comparison of present method and Kuthadi method

\begin{tabular}{||c|c|c|c|c|c|c||}
\hline \hline & $\begin{array}{c}\text { No. of } \\
\text { vehicles }\end{array}$ & $\begin{array}{c}\text { Kutadi's } \\
\text { detected } \\
\text { vehicles }\end{array}$ & $\begin{array}{c}\text { Kutadi's } \\
\text { missing } \\
\text { vehicles }\end{array}$ & $\begin{array}{c}\text { Kutadi } \\
\text { Performance } \\
\%\end{array}$ & $\begin{array}{c}\text { Present Method } \\
\text { detected } \\
\text { Vehicles }\end{array}$ & $\begin{array}{c}\text { Present Method } \\
\text { Performance \% }\end{array}$ \\
\hline Road 1 & 21 & 12 & 9 & 57.1 & 19 & 90.5 \\
\hline Road 2 & 14 & 10 & 4 & 71.5 & 13 & 93 \\
\hline Road 3 & 41 & 32 & 9 & 78 & 39 & 95.1 \\
\hline Road 4 & 9 & 9 & 0 & 100 & 9 & 100 \\
\hline \hline
\end{tabular}

\section{References}

[1] Yamazaki, F., Liu, W.: Vehicle Extraction And Speed Detection From Digital Aerial Images., IGARSS 978-1-4244- IEEE (3-08-2008)

[2] Juozapavicius, A., Blake, R., Kazimianec, M.: Image Processing in Road Traffic Analysis. Nonlinear Analysis: Modelling and Control (2005)

[3] Masakatsu, H., Toshio, H., Kouhei, T.: Traffic Queue Length Measurement Using an Image Processing Sensor (2005-12-10)

[4] Wai, H.L., Lindsay, K.: Real Time Object Tracking using Reflectional Symmetry and Motion. IEEE, Los Alamitos (2006)

[5] Gonzalez, R.C., Woods, R.E.: Digital Image Processing, 2nd edn. Prentice Hall, New Jersey (2002)

[6] Gonzalez Rafael, C., Woods Richard, E., Eddins_Steven, L.: Digital Image Processing Using MATLAB (Hardcover), 1st edn. Prentice Hall, Englewood Cliffs (2003)

[7] Hinz, S.: Automatic Road Etraction in Urban Scenes and Beyond (2005)

[8] Geoffrey, A.: Hollinger, Design and Construction of an Indoor Robotic Blimp for Urban Search and Rescue Tasks (2005)

[9] Zheng, H.: An Artificial Immune Approach for Vehicle Detection from High Resolution Space Imagery (2007) 
[10] Arpad, B., Heipke, C.: Artificial Neural Network for The Detection of Road Junction in Aerial Images, ISPRS Archives. Part 3/W8, Munich, September 17-19, vol. XXXIV (2003)

[11] Erhan, B.: Road And Traffic Analysis from Video. In: A Thesis Submitted to the Graduate School of Engineering for the Degree of Master of Science, August 2007, Koc University (2007)

[12] Rosenbaum, D., Charmettea, B., Kurza, F., Suria, S., Thomasa, U., Reinartza, P.: “Automatic Traffic Monitoring From An Airborne Wide Angle Camera System. In: The International Archives of the Photogrammetry, Remote Sensing and Spatial Information Sciences, Beijing, vol. XXXVII. Part B3b (2008)

[13] Stefan, H.: Automatic Road Extraction in Urban Scenes-and Beyond, Remote Sensing Technology, TU München (2004)

[14] Hu, J., Razdan, A., Femiani, J.C., Cui, M., Wonka, P.: Road Network Extraction and Intersection Detection From Aerial Images by Tracking Road Footprints. IEEE Transactions Geoscience and Remote Sensing 45(12), 4144-4157 (2007)

[15] Kim, Z., Malik, J.: High-Quality Vehicle Trajectory Generation from Video Data Based on Vehicle Detection and Description. In: Proc. IEEE Intelligent Transportation Systems Conference, pp. 176-182 (2003)

[16] Zhenfeng, Z., Hanqing, L., James, H., Keiichi, U.: Car Detection Based on Multi-Cues Integration. IEEE, Los Alamitos (2004)

[17] Albert, B., Steger, C., Mayer, H., Eckstein, W., Ebner, H.: Automatic Road Extraction, German in Photogrammetrie, Fernerkundung, Geoinformation (PFG), No. 1/99 (1999)

[18] Smith Mike, J., Chandler, J., Rose, J.: High Spatial Resolution Data Acquisition for the Geosciences: Kite Aerial Photography. Earth Surface Processes and Landforms (2008)

[19] Hong, Z., Li, L.: An Artificial Immune Approach for Vehicle Detection from High Resolution Space Imagery. IJCSNS International Journal of Computer Science and Network Security 7(2) (February 2007)

[20] Sumalatha, K.: Detection of Object from High-Resolution Satellite Images, A Thesis Submitted to the Graduate School of Engineering for the Degree of Master of Science. University of Minesota Duluth, USA (May 2005)

[21] Serra, J.: Image Analysis and Mathematical Morphology, vol. 2. Academic Press, New York (1988)

[22] National Geographical Organization, http: / / www.ngo-iran.ir/Dphoto/index.html

[23] NAVTEQ Satellite, http: / /www . navteq. com/ 